\title{
Creating Value Through Enterprise Risk Management
}

Frantz Maurer, University Montesquieu-Bordeaux IV (I.R.G.O.) \& BEM Bordeaux Management School, France

\begin{abstract}
The traditional risk management approach has been characterized as a highly disaggregated method of managing financial risks. Recently, risk management has evolved from a narrow, insurance based view to a holistic; all risk encompassing view, commonly termed Enterprise Risk Management (ERM). Financial risks are inherent in financial markets and their management represents one of the main tasks in the business of financial institutions. Enterprise Risk Management enables management to effectively deal with uncertainty and associated risk and opportunity, enhancing the capacity to build value. In contrast to the existing finance literature, this paper emphasizes the practical issues related to the adoption of an ERM framework for strategic decision-making in banks. The aim is to provide an extensive guide to the implementation issues faced by banks that are in the process of implementing fully integrated risk management systems and capabilities.
\end{abstract}

Keywords: Enterprise Risk Management, risk aggregation, risk taking, risk-conscious culture, risk metrics, economic capital.

\section{INTRODUCTION - THE EMERGENCE OF ENTERPRISE RISK MANAGEMENT IN BANKS}

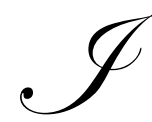

$\mathrm{n}$ the recent years, banks the world over are reevaluating how they address risk. High-profile financial failures over the past decade and an accompanying wave of new regulations - such as Basel II, International Financial Reporting Standards (IFRS) and Sarbanes-Oxley - are driving banks to take a more comprehensive approach to risk management.

The increasing sophistication in the assessment of different risks has resulted into a fragmentation of the different risk disciplines (for example, market risk and credit risk). One reason is due to the fact that risk management process has been driven by independent analyses of different types of risk and the analytical approaches that are subsumed in each of these analyses are complex, difficult and not easily communicated to nonspecialists. Risks are still not dimensioned in similar ways. Although relatively efficient when it comes to assess one specific risk, the risk management process has some difficulties to link well with the business imperatives and the strategy of the bank. And when it comes to select appropriate levels of each risk and selecting or at least articulating an appropriate level of risk for the bank as a whole, the risk management process is doing very poorly.

At most banks, risk management systems are fragmented across organizational silos. Data quality is poor, and measurements are typically inadequate. With inconsistent methods and unconsolidated reporting, banks struggle to manage risk on a companywide basis. As a result, there is a real danger that risk is being inadequately factored into business strategy and capital allocation decisions.

Therefore the main problem that banks face today is how aggregate the different risks of their firm in a way that is meaningful for the decision-maker. The emergence of enterprise risk management (ERM) concepts aims to solve the problem. ERM has rapidly emerged as the new paradigm for managing the complex portfolio of risks facing an enterprise (Tufano, 1996; Liebenberg and Hoyt, 2003; Beasley et al., 2005; Slywotzky and Dzik, 2005). Basically, ERM benefits firms by decreasing earnings and stock-price volatility, reducing external capital costs, increasing capital efficiency, and creating synergies between different risk management activities (Miccolis and Shah, 2000; Cumming and Hirtle, 2001; Lam, 2001; Meulbroek, 2002). 
As we will see in this paper, good ERM practices are hard to find. This may due to the fact that the role of risk management for organization has broadened and as a consequence a new mindset for ERM requires a set of concepts and approaches that fully embraces complexity and ambiguity and that allow for a more balanced view of the risks and opportunities in the business environment. What also seems to be lacking is the backing of the Board and top management level in tackling the task of risk management in a steady, deliberate and comprehensive manner.

But it seems that there are even more fundamental reasons why ERM has not been very successful. These reasons relate to the philosophy of ERM and the way banks have implemented it. One definition that is commonly used describes ERM as the "holistic approach to measuring and managing major risk types based on their simultaneous consideration (and interrelationships when appropriate), thus allowing an institution to understand and adjust its risk exposures in overall risk/reward framework".

For the most part, banks recognize the need to improve risk management capabilities and are moving to address the situation. They have started to invest significantly in integrating different risk types into one framework. In this paper, I suggest that the following points to be considered when adopting an ERM framework:

- $\quad$ Moving beyond compliance

- Delivering value in a changing risk environment

- $\quad$ Adopting a more balanced view of risk and opportunities

\section{MOVING BEYOND COMPLIANCE}

While compliance will remain the focus of risk management efforts in the near term, leading banks are transforming risk management into a full-fledged partner that provides value to the business. The goal is to achieve an integrated, comprehensive and strategic risk program. To achieve this goal, banks must address the need for change on several fronts.

\subsection{Banks Need To Instill A Risk Culture Throughout The Entire Organization}

For most banks, risk management is still not an integral part of business decisions. As a result, risk is inadequately reflected in business strategy and capital utilization. There is no doubt that ERM is an important tool in optimizing shareholder value in the long term. However, it is also important to recognize that any strategic decisionmaking relies on many different types of trade-offs (e.g. growth versus focus on profitability). Properly implemented, an ERM framework should ensure that a desired level of profitability is sustained while maintaining the right level of capital against unexpected losses. Isolated from the other levers that create value (raise performance, maintain growth, gain market share), an ERM framework can lead to the wrong strategy.

It is well known that growth is important for getting shareholders and market recognition. But after some stages, profitability has limits especially when significant effort has been already made to reach high level of returns and efficiency. In this search for growth, a line of business with high growth potential but with a current low RAROC $^{1}$ - relative to other business lines- should not always lead to a reduction of its capital and risk appetite, somehow synonym of reduced business activity in the future. Obviously this search for growth should rapidly transform into profitable growth over a medium term horizon. Therefore in some occasions it is important to ensure high level of profitability and allocation of risk that search for new opportunities of growth, even though with low margin initially. A good understanding of the current and future market dynamics becomes a key element of the formulation of the firm bank strategy. Too much emphasis on profitability can therefore deteriorate value over a certain period.

With ERM, the firm is concerned about creating value for its shareholders. It maximizes firm value by optimizing the tradeoff between risk and return (Nocco and Stulz, 2006). When it comes to shareholder value optimization, it is not clear that focus only on return on capital is the ultimate driver. Indeed there may be good

\footnotetext{
${ }^{1}$ Risk-adjusted return on capital. RAROC is the return earned by a business unit on the capital assigned to it.
} 
reasons for a not very profitable business line to maintain its level of activity and investment. Recently, a leading investment bank decided to grow significantly their market share in the commodities market. Their profitability in recent years did not match management expectations but nonetheless their focus has remained the same. Moreover, they are even considering increasing their level of capital expecting higher market volatilities in the future. In that case, the bank has recognized that building its reputation in this new activity will take time and will unlikely get high returns in the short term.

For ERM to become an integral part of business operations, the bank's risk management strategy must align with and actively support its business strategy. For example, risk management should influence which customer segments are targeted and how particular segments are served. Although this linkage between customer relationship management and risk has not been fully established in all firms and lines of business, some banks use sophisticated risk management techniques in specific business areas such as pricing mortgage loans. Taking into account the full credit correlation between different customers across their portfolio allows the bank to boost profitability because it can provide more competitive prices for some of its target loan products while still achieving the required overall return on capital.

\subsection{Banks Need Sound Risk Metrics For Building A Risk Culture}

The vast majority of banks struggle to quantify the benefits of their risk management efforts. By and large, banks lack the appropriate key performance indicators for assessing individual or business performance. Developing the right metrics can enable banks to assess Chief Risk Officer performance quantitatively, based, for example, on how effectively they avoid losses or optimize the trade-off between risks and rewards.

The adoption of an ERM approach will be mainly based on the trust of the different stakeholders on the number produced by the model. If the decisions that are taken are not perceived as fair, it is unlikely that large organizations will adopt such model. There are many examples of organization transformation that have failed due to lack of faith in the assumptions of that change. Suspicion in the numbers may come from different sources.

For example, it is well known that if markets suddenly experience severe turbulence, a risk measure such as Value-at-Risk (VaR) will inevitably underestimate the risk and will only capture the sudden jump in volatility a few days down the road (Samanta et al., 2005). VaR lacks an important mathematical property referred to by Artzner et al. (1999) as subadditivity. Intuitively, subadditivity is associated with the notion of risk reduction through diversification. One important reason why $\mathrm{VaR}$ is not a robust risk measure is that it does not always provide an accurate sense of degree of diversification within a portfolio.

Moreover, market Value-at-Risk methodologies assume that returns follow a geometric Brownian motion and are iid (identically and independently normally distributed). However, many empirical analyses of financial returns have demonstrated that the assumption of independence is not supported: the size (but not the direction) of yesterday's return does not have implication for today's return. A large return yesterday is likely to be followed by a large return in either direction. This phenomenon is called volatility clustering and is only one example of potential source of risk model inherent to current risk metrics and models in place. Other source of model risk includes the assumption of normality of returns, the fact that the mean and the variance remain constant over time, instability of the correlation measures, etc. Although many progresses have been made (e.g. use of GARCH model) for volatility estimation or the use of normal mixture or Student distribution to rectify such drawbacks, they are not easy to implement due to either lack of data or computer performance related issues.

\subsection{Banks Need To Drive Acceptance Of ERM Across All Business Units}

Risk initiatives require active cooperation among the business lines heads that will ultimately benefit from risk done right, and the top management who define the firm's risk appetite and policies. The Economist Intelligence Unit (2005) reports that many organizations appoint a Chief Risk Officer or CRO to oversee the enterprise's risk management process. Walker et al. (2002) notes that because of its scope and impact, ERM requires strong support from senior management. 
Line managers are often the best sources of information on factors that should be incorporated in risk management policies, such as market and competitors trends, and customer needs. But many business managers do not fully understand or are not fully committed to risk management disciplines. While risk managers and line-ofbusiness managers agree on the desired strategic role of ERM, the latter are more inclined to see risk management as an "as and when needed" function. However, only by integrating risk management into the business decisionmaking process can a bank make solid decisions.

In recent years, the risk management function has been more centralized with the creation of a group risk function. The main purpose of this organizational response was to empower one individual or group with the responsibility to evaluate overall firm-level risk, and determine the best interest of the bank as a whole. Current thinking is to reverse the process of centralization. There are different reasons for that.

The first reason is that banks are still very much organized in silos and heads of divisions don't like having power taken away from them. The second reason is due to the lack of robustness of a firm-wide risk methodology that are still unable to take into account properly correlation and diversification effects between the different risk types. The third one is the limitation of technology and the difficulty to aggregate consistently all the risk information at a central level. For example, very few banks still do not have a single view of customer risk which is the basis of robust counterparty risk management practices. Fourth, by creating a new risk function at the center, there is a possibility that it results in extra cost and generates duplications thus reducing the overall efficiency of the risk function in an environment where cost pressure is still very high.

\subsection{Banks Need Accurate And Real-Time Risk Information}

A key tenet of sound risk management is risk transparency, both in terms of internal risk reporting as well as external disclosure (Lam, 2007). Real-time risk information is lacking, unconsolidated or too aggregated. While there is great demand among business unit managers for automated, consolidated reports and "risk snapshots" as needed, few banks are able to satisfy it today. The ability to generate reports much more frequently, every day or even in real-time, would make risk management a much more flexible, powerful and valued tool for business managers. For example, while banks typically provide market and counterparty risk reports on a monthly or weekly cycle, risk managers indicate they would like to receive them daily or even in real-time. The ability to generate reports on a real-time basis would make ERM a much more flexible, powerful and valued capability for business managers.

As more performance and risk information are made available, Asian banks should develop dashboard reporting to enhance the delivery of such information to key decision makers. In other words, the purpose of dashboard reporting is to enhance the "risk intelligence" of the bank (Lam, 2007). Managers and external stakeholders are increasingly demanding clearer and more standardized reporting in order to better understand the risks and compare levels of risk across business units or different firms. Designing effective enterprise risk reports is the foundation for clear and timely communication of risk across the bank. Risk reports must be produced and distributed to many individuals and businesses. The format and content of these risk reports must be designed to suit the specific needs of each organization level. Risk reports at each level of the organization show whether risks are taken within prescribed internal limits. Risk reports are also useful for evaluating risk-adjusted performance.

Relevant risk indicators must be reported to all stakeholders, providing the information needed to make decisions at business unit and firm levels. ERM strategy and risk indicators must be communicated on a regular basis to all levels of the bank. Decision makers cannot manage what they cannot see. They need ready access to risk information to respond appropriately to threats and opportunities as they arise. Risk should be aggregated/disaggregated in a coherent manner across a bank, making risk reporting much more flexible and informative for top management. The ability to generate reports on a flexible basis would make ERM a much powerful capability for business managers. 


\subsection{Banks Need An Enabling Infrastructure}

The demands of regulatory compliance are driving IT costs upward. As a whole, however, banks have failed to realize the full benefits of their IT investments and have dedicated insufficient resources to developing and improving risk management. As a result, processes, systems and capabilities remain fragmented or - worse incompatible. An integrated risk management IT architecture with centralized data warehousing can deliver more efficient and effective reporting.

As technology advances, the possibilities increase. For example, because of its ubiquity, the Internet can provide better and faster ways of disseminating information and risk analysis and conducting risk transfer transactions. Increased computing power and continually declining storage costs make more refined analytics possible and more practical, which opens sophisticated risk models to mid-sized companies. The same computing power also makes it possible to measure and report on risk more frequently - even in real-time.

Most analysts and experienced practitioners admit that the best solutions for long-term and cost effective risk management involve the use of information technology. For example, Goodwin (2005) highlights that software tools can help businesses steer through the minefield of meeting compliance regulations and offer real cost savings. ERM software solutions increase the potential for present and future system integration with other applications such as ERP, and enable on-going knowledge retention and sharing (Hewitt, 2005).

\section{DELIVERING VALUE IN A CHANGING RISK ENVIRONMENT}

With the right strategic approach, banks can transform their ERM function, control costs and move toward making risk management a full-fledged, valued partner to the business units. Three strategies can help banks transform their risk management activities into a full-fledged ERM capability.

\subsection{Improve Data Quality And Availability}

Most banks recognize the need for improved data. While regulatory changes have highlighted the need to improve data quality and to develop audit and control trails for risk management reporting, data management remains a significant barrier for implementing ERM strategies. Clearly, ERM can only be as good as the data that underpins it. Thus, a major challenge for banks is to improve data quality by integrating data capture at its source. As stated by Schlottmann et al. (2005), a main obstacle to integrated risk management is data problems ${ }^{2}$. Due to insufficient data, as in the area of operational risk management for example, statistical methods fail to deliver clear statements. Correlations also emerge in the context of inadequate data supplies. If there is only short historical data available or if the parameters prove to be highly volatile empirically, found correlations often fail.

Data systems must be integrated and facilitate automatic collection and consolidation of data to help prevent errors and improve currency. Standard data models and data formats can help banks achieve the consistency required for reliable decision making. However, implementing these standards is not simple, and can be very expensive as many banks have found. Successful banks tend to break the problem down into manageable chunks. Instead of implementing one central- all-encompassing data warehouse with a single standard system interfacesome firms choose to implement a series of mini-warehouses or data marts, each specialized in the date required for a single function. Although this approach increases the number of interfaces and introduces the need to reconcile data across data marts, reliable tools exist to synchronize data and prevent erroneous information from ever entering the database in the first place.

\subsection{Manage Risk Across The Enterprise}

To be effective, ERM must satisfy three requirements. It must be integrated (spanning all lines of business), comprehensive (covering all types of risk) and strategic (aligned with the overall business strategy).

\footnotetext{
${ }^{2}$ Similar terms are consolidated risk management or enterprise-wide risk management (cf. Cumming and Hirtle, 2001).
} 
The rationale for the integration of market, credit and operational risk has got different sources. First, the determinants of market risk are a subset of those of credit risk as both counterparty exposure and market positions are determined by the composition of the distribution of similar market factors. The main argument for integrating the measurement of operational risk with that of credit risk and market risk is mainly based on a commonality of statistical techniques. Also, clear correlations exist between credit risk and operational risk. A classic example is around the legal documentation attached in credit derivatives transactions where misinterpretation of a legal element of the deal may change radically its risk profile. The idea that different risk types are correlated and should be offset is a great idea but as a practical matter, it has proven very difficult to implement.

The core of such an integrated risk management system is represented by an appropriate risk aggregation methodology. In the Basel Committee report (2003) this is explained as follows: "Broadly, risk aggregation refers to efforts by firms to develop quantitative risk measures that incorporate multiple types or sources of risk. The most common approach is to estimate the amount of economic capital that a firm believes is necessary to absorb potential losses associated with each of the included risks."

Although correlations do exist amongst these different risk types, they are very difficult to measure due to lack of historical evidences. Operational events have only started to be collected some years ago and for some major disasters events, very few evidences are available (grouped in the famous "high impact low frequency" category). As a result, most if not all of these risk models do not view this array of risks as a standard portfolio problem. Rather, they separately evaluate each risk and aggregate total exposure by simple addition. As a result much is lost in the aggregation procedure. Moreover, the analytical approaches that are subsumed in each of these analyses are complex, difficult and not easily communicated to non-specialists. Two theoretically sound approaches consist in the so-called top down and bottom up approaches. Both approaches are a step towards an enterprise-wide risk management framework, which can support management decisions on an enterprise-wide basis by integrating all relevant risk components (Grundke, 2006). In practice, some kind of heuristics, based on strong assumptions, are often used to merge the economic capital numbers for the various risk types into one overall capital number ${ }^{3}$.

Driving fundamental change in the risk management function requires a comprehensive approach. Successful banks will implement a framework of activities and enablers - including infrastructure - for ERM. Such a framework consists of a comprehensive group of activities that can help banks manage risk from a portfolio perspective, recognizing the diversity of risks and the mitigating or multiplicative effect they have on each other. These activities fall into three categories:

Risk control - Policy development, risk assessment and risk monitoring

Risk management - Reporting and risk mitigation

Business management - Decision making and monitoring

To accomplish these activities, banks need a core set of enablers including the right organization, accurate data and the capability to capture and analyze it, effective stakeholder management and, perhaps most importantly, a corporate culture where managing risk is part of the daily routine.

This type of framework provides a clearer view of the combined effect of enterprise risks and can help turn decision makers in all parts of the business into better-informed risk managers. As a result, the risk management function can offer clear and balanced views of both threats and opportunities and contribute significantly to better business results.

As shown in Figure 1, an ERM framework consists of both activities and enablers. Activities are performed by a centralized risk management function as well as by the business units themselves, and range from top-level strategy development to everyday decision making. The enablers are supporting elements that allow the business to accomplish the desired activities. They include both tangible and intangible elements.

\footnotetext{
${ }^{3}$ Rosenberg and Schuermann (2006) provide an excellent overview on risk aggregation methods used in practice.
} 
ERM begins with the development of a risk management strategy that is aligned with overall business goals. Policy development, risk assessment and risk monitoring should be based on the bank's overall strategy, the risks and potential risks it faces and the key risk indicators across the bank's units.

ERM helps lower the cost of risk transfer through a portfolio approach and through transparent management of overall risk. Reporting should be driven by a clear understanding of organizational policies and industry regulations, while risk mitigation should be guided by criteria for evaluating, responding to and transferring risk. Business management should focus on decision-making and monitoring, with an emphasis on risk reduction and avoidance, business controls and compliance.

Finally, banks can drive their ERM capability to full maturity by developing risk management as a partner with the rest of the organization in mergers and acquisitions, active portfolio management, pricing of complex products and other activities.

\section{Figure 1. A framework that promotes integrated, comprehensive and strategic ERM.}
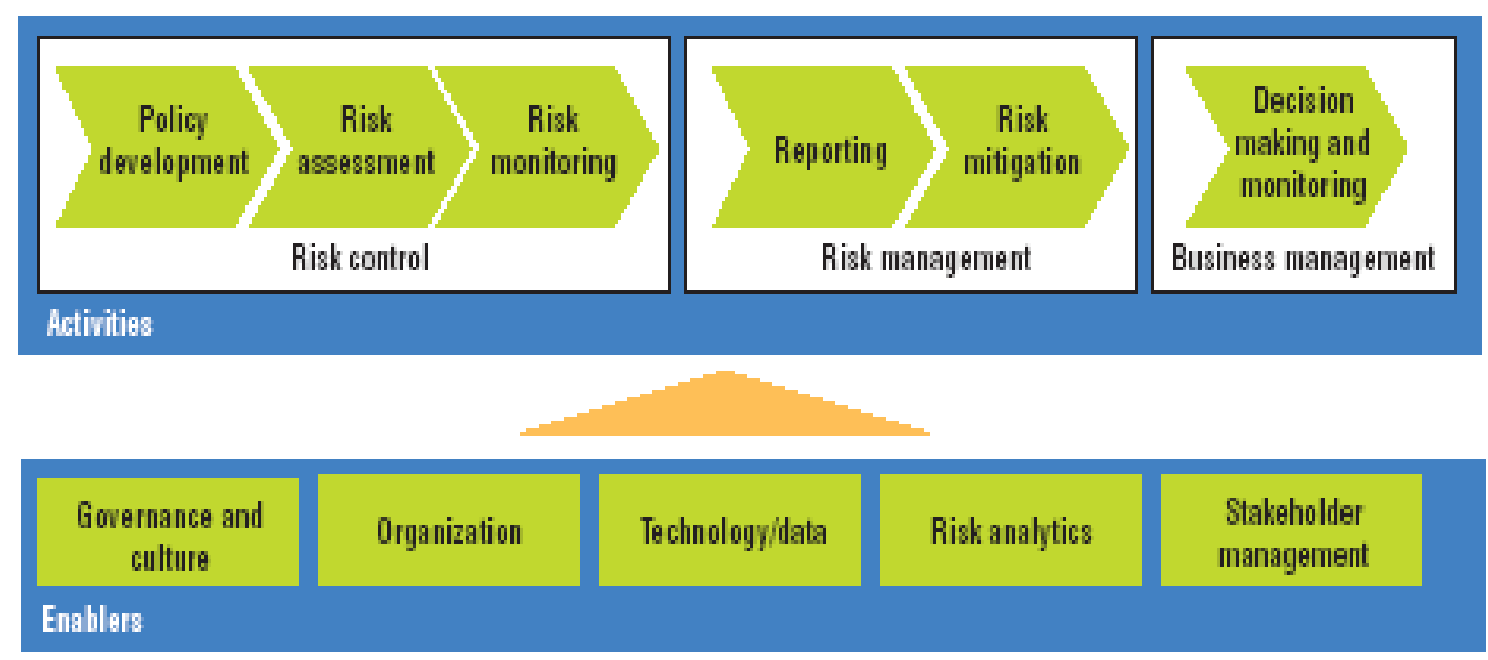

Enterprisewide portfolio approach

- Risk is considered from an enterprisevide or porffolio perspective recognizing the dinersity of risks and mitigating measures and their effects on the enterprise risk tolerances

- Portfolis management provides a direct link between risk management and sharehobler value

Sources: "Enterprise Risk Management. From Incentives to Controls," James Lam, Wiley Finance, 2003 \& IBM Business Consulting Services, Risk and Compliance Practice, 2004.

\subsection{Promote The Softer Side Of Risk Management}

It is now widely recognized that there is much more to risk management that just risk measurement. Clearly, financial risks and their management represent one of the main tasks in the business of banks. As a result, the possible extents of these risks have to be quantified. As this involves high uncertainties, Alexander and Pézier (2003) use the term risk assessment rather than risk measurement. But, perhaps too much focus has been placed on the sophistication and apparent precision of risk estimation models, and not enough on the more important managerial and judgmental elements of a strong ERM, that is the softer side of risk management. Risk measurement is but an intellectual exercise unless it is used actively in managing businesses. Banks should adopt ERM as the new form of corporate governance and a better business practice. The announcement of a new Chief Risk Officer (CRO) is a signal that the firm is establishing an ERM program. Whereas earlier announcements concentrated on the CRO's role as risk champion, several of the latest announcements place greater emphasis on the CRO's responsibility and accountability for good corporate governance (Liebenberg and Hoyt, 2003). 
In the 90s, a common methodology to transform each risk types at a firm-level was needed. The emergence of Value-at-Risk (VaR) and Earning-at-Risk (EaR) techniques has allowed integrating first market risk and then crediting risk across the enterprise. These approaches have been very popular (and still are) and considered as the best way to unify heterogeneous methodologies within each risk category into a single framework.

Beware of rushing into risk analytics or applying checklists. Even the most sophisticated risk measurement technology will not meet stakeholders' objectives if mechanically adopted without managerial judgment. Creating a culture that embraces ERM will result in risk management decisions that increase economic value for a chosen risk appetite and time horizon.

For today's banks, moving beyond compliance to enhanced business value requires a firm wide ERM culture grounded in relevant metrics. With the right metrics in place, top management can show benefits as part of a communication program to improve the perception of risk management. One way to drive adoption of the risk management culture is to clearly communicate how ERM adds value for the lines of business. Top management must recognize value of risk management and actively encourage employees to incorporate ERM in their work. The key is to engage in an ongoing dialogue, proactively assisting the business units with the decisions they face while continuously communicating how ERM leads to better decision-making, which in turn, benefits the business units and the bank alike. Communication must be an integral part of the objective of an ERM team, stressing the importance of explaining the benefits of ERM to the business lines.

Beyond the physical constructs that can encourage enterprise-wide risk management, some enablers relate more to the prevailing attitudes and conduct of the organization itself. For example, banking industry leaders integrate ERM into their corporate governance and promote a risk-conscious culture. Simply put, ERM becomes ingrained in the moral constitution of the bank. Banks can grow a strong risk culture through appropriate governance, communication and management of expectations, both within the bank and externally. As recently noted by Pagach and Warr (2007), firms have implemented ERM for what might be considered to be non-risk management reasons. These authors found some evidence that firms that have seen recent poor stock performance are more likely to hire Chief Risk Officers - a result consistent with firms trying to demonstrate a credible response to a period of poor performance.

ERM is re-entering organizational life as a demand for decisions in areas where some pretense of knowledge is a necessary defense against anxiety. The appearance of manageability is created by a material abundance of standards and metrics. However, an "intelligent" business management approach would not allow stamping managerial attention and independent critical imagination. Ultimately, the real danger is to displace valuable professional judgment in favor of defendable techniques and models.

\section{ADOPTING A MORE BALANCED VIEW OF RISK AND OPPORTUNITIES}

Sophisticated risk measurement systems and methodologies are of non avail if they are not carefully and fully integrated with the capabilities and ambitions of the bank. Using an ERM framework allows management to determine clearly their risk appetite, set profitability targets and decide on priority lines of business.

\subsection{Linking Economic Capital And Risk Appetite}

Economic capital management frameworks can assist a bank in understanding and managing its overall risk profile. Bank's risk appetite must be clearly defined and guides economic capital allocation. Instead of focusing only on maximizing revenue, banks should consider return on risk in strategic planning and budgeting. Clearly, the risk taken should relate business performance evaluation. Banks should link compensation of risk takers to their return on risk, as opposed to their absolute returns. Risk limits should follow a firm's hierarchical organizational structure and set expectations for risk taking and profitability. Risk-based limits empower managers to allocate economic capital dynamically to areas that offer the best opportunities, or in which the bank's expertise is the greatest. Senior management must be responsible for the monitoring of risk taking with regard to risk limits, and make sure that risk limits consistently reflect the bank's risk appetite, overall business strategies and financial resources. 
It is important to assess the level of capital to sustain risk-generating activities and relating the cost of capital to the riskiness of business activities or projects. Clearly, the level of risk taken should relate to capital (more risk $=$ more capital). If a sensible link exists between risk and capital, banks can set minimum threshold return on capital targets, and optimize risk adjusted performance by maximizing return on capital. Conceptually, economic capital is defined as a cushion for unexpected losses. It ensures that an organization remains solvent and stays in business even under extreme conditions. Banks need an ERM capability to ensure that their returns (profits) outweigh the risks that they are running. They must determine an efficient economic capital for their business. Too little capital puts a company at risk of financial distress because of potential adverse market fluctuations. Setting aside a lot of capital is safer, but costly and inefficient. Finding an optimal capital structure involves finding the right balance between the need for safety and the desire for maximizing return on capital.

Adhering to the principles of economic capital lets financial institutions constantly reassess their investment strategies and make improved operational decisions regarding pricing and capital allocation. With the ability to determine their own risk appetite and manage resources against this metric, banks can strike a balance between risk and returns for the company, and achieve the optimal results within the defined regulatory constraints (e.g. Basel II).

Ultimately, the resulting economic capital attributable to each business unit or line of business provides a framework that allows management to understand and evaluate sustainable performance and manage company portfolio via a common, neutral metric. As a consequence, it seems that banks are thus better able to improve shareholder value by reallocating capital to business units that generate strategic value and sustainable returns, or that demonstrate long-term growth and profitability - on an objective and economically sound basis.

\subsection{Is Risk Bad For Business?}

Most often, risk is based on coping strategies that tend to avoid risk. Such defensive approach - although needed-relies on sophisticated mathematical models that we know are not totally reliable (who can predict the future?). This approach largely promoted by the regulators has got its limits. Not only it is impossible to control everything (remember Long Term Capital Management, the hedge fund mainly built around complex mathematical models that came close to collapse in 1998) but more importantly it may limit and constrain banks ability to grow and take advantage of significant business opportunities.

Risk is not always bad for business and there is a real need to develop a bigger picture of risk management that balances risks and opportunities. Increasingly, organizations have come to recognize the opportunistic side, the value-creating potential of risk. Risk-taking is necessary for growth and success and the new risk management approach should re-embrace risk as a source of advantage. In essence, there is a realization that risk is not completely avoidable and, in fact, informed risk-taking is a means to competitive advantage (Casualty Actuarial Society, 2003). To do this, it is therefore critical to reinforce the link between risk and strategy through the definition of the risk appetite of the bank.

The classic separation between the risk takers and the risk controllers overlooks the fact that these former opportunity-minded professionals manage risk too, just in a different manner. Banks need to integrate the risk-taking and the risk controlling sides and involve all the different views and perspectives within the organization (business executives, heads of business lines, risk managers) as well as including the perceptions of shareholders, customers, regulators and other external parties into the equation.

To accomplish both objectives- linking better risk and strategy and integrating the risk-taking and the risk management sides together- banks need to adopt a fundamentally different approach. The first step of this approach is to articulate a plausible future state that is linked to the different strategic initiatives that have already been agreed. The plausible future state is directly derived from explicit assumptions made by the top management about the future. Making very explicit what are the possible threats and opportunities allows to management to identify risks in the context of their strategy and the possible opportunities. 
The second step consists of creating the different scenarios that are directly derived from the different plausible future state. Scenarios could be grouped along different dimensions (for example, likelihood of happening or overall impact on the bank). Heads of business lines and risk experts jointly challenge perceptions and preconceived ideas about the future and the types of risk and opportunities. An inventory of what is known about the future is developed and, based on a rigorous process, a number of future (not probable) scenarios are designed.

The third step is to develop a scenario tree to allow stakeholders to assess the impact each scenario could have on strategic success. By allowing for scenarios that can impact success in a positive or negative direction, the scenarios tree restores the balance of risk and opportunity to overall risk management. Risk and opportunities can be then grouped in many different ways. For example, risk can be divided between strategic risks (those that are taken in pursuit of a strategic goal) and accidental risks (those that have a downside only and are directly linked to a given strategy). In this context, accidental risks can be considered as an overhead cost of doing business.

Finally, the last step consists of monitoring each strategic initiative and track the evolution and progression of the contextual conditions. The focus is on monitoring the key factors that affect the realization of a scenario rather than its underlying risk factors. The evolution of the scenarios is assessed using business skills and experience backed by econometric analysis of macro-economic variables that determine the context of the scenario. This requires a new mindset with a capacity for learning, appreciation of ambiguity and uncertainty, and recognition of the value of strategic conversation.

With this approach, decisions are being made by considering both sides of the same coin, the downside and the upside of risk. This approach has also the advantage to simplify significantly the ERM process as it reduces the universe of various risks to a manageable numbers (around fifty). Also, it re-emphasizes the need for judgment and imagination in organizational life as a demand for decisions in areas where some pretence of knowledge is currently considered as a necessary defense against anxiety.

\section{CONCLUSION - SHIFTING PRIORITIES}

This paper has discussed some of the key issues related to the adoption of an Enterprise Risk Management framework. Many banks have already experienced some difficulties in the adoption of their ERM system and are still learning about the interaction of ERM and strategic decision-making. This paper has outlined how a comprehensive risk management framework can help banks upgrade risk management from a necessary burden to a highly-valued and well-integrated business discipline. While ERM provides important benefits, limitations exist. They result from the realities that human judgment in decision making can be faulty, and breakdowns can occur because of human failures such as simple errors or mistakes (Committee of Sponsoring Organizations of the Treadway Commission, 2004).

When it comes to Enterprise Risk Management, banks have got a unique opportunity to think differently and implement a new approach that is more aligned with business objectives. Traditional risk types tend to rely too much on statistical results and too little on personal judgment and knowledge of the business. The appearance of manageability is created by a material abundance of standards and metrics. However, an "intelligent" risk management approach should not allow swamping managerial attention and independent critical imagination. Ultimately, the real danger is to displace valuable professional judgment in favor of defendable techniques and framework. More importantly perhaps is the fact that risk becomes at the core of any strategic decisions.

\section{AUTHOR INFORMATION}

Frantz Maurer holds a Ph.D. in Business Administration from the University of Bordeaux in France. He is currently an Associate Professor in finance both at the University of Bordeaux and BEM Bordeaux Management School. He is also an Adjunct Lecturer at HEC Paris. Frantz is the author of several publications directed to academics and practitioners on risk management. His current research interests focus on Enterprise Risk Management for financial institutions, operational risk in banks, and risk management practices in hedge funds. 


\section{REFERENCES}

1. Alexander, C., and Pézier, J. 2003, “Assessment and Aggregation of Banking Risks", ISMA Centre, University of Reading, UK, $9^{\text {th }}$ Annual Round Table of the International Financial Risk Institute.

2. Artzner, P., Delbaen., F., Eber., J-M., and Heath., D. 1999, "Coherent Measures of Risk", Mathematical Finance, 203-228.

3. Basel Committee on Banking Supervision: Trends in Risk Integration and Aggregation. 2003, Basel Committee on Banking Supervision.

4. Beasley, M.S., Clune, R., and Hermanson, D.R. 2005. "Enterprise Risk Management: An Empirical Analysis of Factors Associated With the Extent of Implementation", Journal of Accounting and Public Policy, 24 (6), 521-531.

5. Casualty Actuarial Society-Enterprise Risk Management Committee. 2003, "Overview of Enterprise Risk Management", http://www.casact.org/research/erm/.

6. Committee of Sponsoring Organizations of the Treadway Commission (COSO). 2004, "Enterprise Risk Management-Integrated Framework".

7. Cumming, C.M., and Hirtle, B.J. 2001, "The Challenges of Risk Management in Diversified Financial Companies", FRBNY Economic Policy Review, 1-17.

8. Economist Intelligence Unit. 2005, "The Evolving Role of the CRO”, The Economist Intelligence Unit, London/New York/Hong Kong.

9. Goodwin., B. 2005, "Software Can Ease the Pain and Cut the Cost of Compliance", Computer Weekly, www.computerweekly.com

10. Grundke, P. 2006, “Integrated Risk Management: Top Down or Bottom Up?" http://www.gloriamundi.org

11. Hewitt., R. 2005, "Enterprise Risk Management: A Long-term Solution for Compliance, Governance and Sustained Growth in Shareholder Value". White Paper, Entropy International, www.entropyinternational.com

12. Lam, J. 2001, "The CRO Is Here to Stay”, Risk Management, April, 16-20.

13. Lam, J. "Enterprise Risk Management at Asian Banks: From Challenges to Strategies". 2007, Executive White Paper, Asia Risk Management Institute (ARMI).

14. Liebenberg, A.P., and Hoyt, R.E. 2003, "The Determinants of Enterprise Risk Management: Evidence From the Appointments of Chief Risk Officers", Risk Management and Insurance Review, Vol. 6, No. 1, 37-52

15. Meulbroek, L.K., 2002. "Integrated Risk Management for the Firm: A Senior Manager's Guide”, Journal of Applied Corporate Finance, 14, 56-70.

16. Miccolis, J., and Shah, S. 2000, "Enterprise Risk Management: An Analytic Approach",http://www.tillinghast.com/tillinghast/publications/reports/Enterprise_Risk_Management_An_ Analytic_Approach/erm2000.pdf

17. Nocco., B.W. and Stulz, R. 2006, "Enterprise Risk Management: Theory and Practice”, Ohio State University working paper.

18. Pagach, D., and Warr, R. 2007, "An Empirical Investigation of the Characteristics of Firms Adopting Enterprise Risk Management”, http://ssrn.com/abstract=1010200

19. Rosenberg, J.V., and Schuermann, T. 2006, “A General Approach to Integrated Risk Management with Skewed, Fat-tailed Risks", Journal of Financial Economics, Vol. 79, No. 3, 569-614.

20. Samanta., P., Azarchs., T., and Hill., N. 2005, "Chasing Their Tails: Banks Look Beyond Value-at-Risk", Enterprise Risk Management For Financial Institutions. Rating Criteria and Best Practices, Standard\&Poor's, 25-31, www.standardandpoors.com

21. Schlottmann, F., Mitschele, A., and Seese, D. 2005, "A Multi-Objective Model Framework for the Integrated Management of Financial Risks", http://www.gloriamundi.org

22. Slywotzky, A.J. and Drzik. J. 2005, "Countering the Biggest Risk of All”, Harvard Business Review, April, 78-88.

23. Tufano, P. 1996, "Who Manages Risk? An Empirical Examination of Risk Management Practices in the Gold Mining Industry". Journal of finance, 51 (4), 1097-1137.

24. Walker, P.L., Shenkir, W.G., and Barton, T.L. 2002, "ERM in practice”, Internal Auditor, August, 51-55. 
NOTES 